\title{
Protracted vomiting as the presenting sign of posterior fossa mass lesions
}

\author{
GONZALO TORREALBA, SERGIO DEL VILLAR, PAULINA ARRIAGADA \\ From the Departments of Neurology and Neurosurgery, Catholic University of Chile, Santiago, Chile
}

SUMMARY Four patients with mass lesions of the posterior fossa experienced protracted vomiting as their only symptom for extended periods of time. The responsible lesions were a cerebellar tumour in two patients, a ventricular cysticercus in one patient, and a giant vertebral artery aneurysm in another. All four cases had compression or displacement of the floor of the fourth ventricle, where the "vomiting centre" has been located. The value of vomiting as a sign of a posterior fossa lesion is emphasised.

Vomiting in association with headache and other neurological signs suggests a central nervous system (CNS) lesion. However, when vomiting occurs in isolation and is protracted, other mechanisms (such as gastrointestinal, endocrine, metabolic, toxic, drugrelated, or psychogenic) are suspected. ${ }^{1}$ We report four patients with protracted vomiting as the presenting sign of otherwise unsuspected posterior fossa mass lesions. The importance of this sign in lesions of the floor of the fourth ventricle is emphasised.

\section{Case reports}

Patient 1 A 55 year old woman had had recurrent vomiting for 3 years when she presented in January 1985. Vomiting was preceded by nausea, and usually occurred approximately 1 hour after meals. Cholecystography showed non-filling of the gall bladder and she underwent an elective cholecystectomy. Vomiting persisted at a frequency of 3 or 4 times per week, and abdominal ultrasound, contrast radiology of the gastrointestinal tract, and laparoscopy were normal. "Psychogenic" vomiting was diagnosed. Vomiting became more frequent over the 8 months prior to admission,

Address for reprint requests: Gonzalo Torrealba, M.D., Hospital Clínico, Universidad Católica, Department of Neurosurgery, Marcoleta 347, Santiago, Chile.

Received 18 November 1986 and in revised form 8 April 1987. Accepted 16 April 1987 occurring in the early morning, frequently related to postural changes, and at times being described as "explosive". She lost $20 \mathrm{~kg}$ in weight over this period. During the last 2 months prior to evaluation, she experienced recurrent dizziness and gait unsteadiness. On neurological examination, multidirectional nystagmus and mild gait ataxia were detected. Computed tomography (CT) revealed a mass lesion in the cerebellar vermis, which at surgery was found to be a Grade III astrocytoma of the vermis, attached to the inferior portion of the floor of the fourth ventricle. Vomiting ceased after operation.

Patient 2 A 29 year old woman presented early postprandial vomiting 3 to 4 times a week during the first and second trimester of her fourth pregnancy. Although vomiting occurred less frequently in the last trimester of pregnancy, it continued after delivery. One month later she started experiencing headaches and was admitted to the hospital in November 1974. General physical and neurologic examinations were within normal limits. She had had a weight loss of $12 \mathrm{~kg}$. Contrast radiology of the gastrointestinal tract and an electroencephalogram (EEG) were normal. Plain skull radiographs showed an enlargement of the sella turcica. A pneumoencephalogram showed no entry of air into the ventricular system, and contrast ventriculography revealed a mass lesion in the caudal aspect of the floor of the fourth ventricle, with associated hydrocephalus. A ventriculoperitoneal shunt was inserted, and a cysticercus cyst firmly attached to the floor of the fourth ventricle was incompletely resected. She became asymptomatic after operation.

Patient 3 A 72 year old man was admitted to hospital in November 1984 because of persistent vomiting. He had been diagnosed as having had episodes of subarachnoid haemorrhage in 1969 and 1977 , but angiography had apparently 
not been performed. During the year prior to admission he had had postprandial vomiting once or twice a week, becoming more frequent during the last month. He had had weight loss of $10 \mathrm{~kg}$. The initial neurological examination, as well as abdominal radiographs and ultrasonography were normal. On endoscopic examination he had evidence of esophagitis and gastric reflux, as well as a $1 \mathrm{~cm}$ duodenal polyp, whose biopsy revealed non-specific chronic inflammatory features. The EEG showed mild diffuse nonspecific slowing. The symptomatology was attributed to the duodenal polyp and he was discharged from the hospital. One week later he was readmitted because of continued vomiting. A second endoscopic examination was unchanged, and the neurological examination remained normal. CT revealed hydrocephalus and a right cerebellar hemispheric lesion, that displaced the fourth ventricle to the left. Following a shunt procedure, a vertebral right angiogram disclosed a partially thrombosed giant aneurysm of the distal aspect of that vessel. The patient transiently improved following the shunting procedure but vomiting reappeared after 1 month, complicated by aspiration pneumonia. A follow-up CT scan showed resolution of the previous hydrocephalus. Following a recurrent pulmonary infection he died in March 1985.

Patient 4 A 50 year old woman was admitted to hospital in November 1982 after a 1 month history of recurrent vomiting after meals. She also reported weight loss (unquantified), anorexia, and malaise. On general physical examination she had a diffuse goiter. Abdominal ultrasound and endoscopy were normal. The diagnosis of hyperthyroidism was established by appropriate laboratory tests. She was treated with propylthiouracil in preparation for thyroidectomy. Vomiting still persisted one and a half months later, and she developed dizziness and gait unsteadiness. Neurological examination revealed multidirectional nystagmus, depressed gag reflex on the right side, gait ataxia, and right cerebellar ataxia. A tumour of the posterior fossa and hydrocephalus were revealed by CT. The patient died suddenly before posterior fossa craniectomy could be performed. Necropsy revealed a medulloblastoma of the cerebellar vermis with slight extension into the right hemisphere.

\section{Discussion}

In our four patients, vomiting preceded the onset of headaches or other neurologic symptoms for a period ranging from 2 months to 3 years, during which time various erroneous diagnoses were entertained. In one patient vomiting was attributed to a duodenal polyp. Another patient was submitted to a cholecistectomy, and after the symptom failed to respond to the procedure, it was considered to be "psychogenic". In another patient vomiting appeared during pregnancy, and a brain lesion was only suspected after it continued following delivery.

All patients ultimately proved to have lesions in the posterior fossa that displaced or compressed the caudal portion of the floor of the fourth ventricle, where the "vomiting centre" is located. ${ }^{2-4}$ The vomiting centre integrates information coming from various nuclei such as those of the area postrema, vestibular and solitary tract nuclei. Anyone of these can be activated by an appropriated stimulus causing vomiting. ${ }^{5}$ A compression or displacement of the vomiting centre or its afferent nuclei or tracts might then explain vomiting in our patients. On the other hand, demyelination of these areas has been reported to cause an absence of vomiting, ${ }^{7}$ as in experimental lesions in the area postrema or the vomiting centre. ${ }^{26}$

On rare occasions, lesions far from the "vomiting centre" can bring about persistent vomiting, as it has been described in a child in whom cyclic vomiting was documented to be the expression of simple partial epilepsy, caused by a thalamic and right temporal tumour. ${ }^{8}$ In a study of 100 epileptic patients with visceral symptoms occurring either as an aura or as an ictal phenomenon, eight patients presented vomiting, occasionally preceded by nausea. Two areas were considered to be responsible for these symptoms: (1) the fronto-temporal region, including the anterior portion of the temporal lobe, the insula, and the orbital surface of the frontal lobe, and (2) the parasaggital portion of the frontal lobe. ${ }^{9}$

It could be argued that vomiting in our patients was simply caused by increased intracranial pressure secondary to the hydrocephalus, but the latter was not present in patient 1 , and in patient 3 vomiting persisted after successful ventriculo-peritoneal shunting. Furthermore, vomiting appeared long before the onset of headache in patient 2 , and no patient had papilloedema.

It has been suggested that neither headache nor vomiting, classically attributed to intracranial hypertension, are actually due to increased intracranial pressure. In pseudo-tumour cerebri or intracranial hypertension induced by the infusion of saline to the subarachnoid space, headache is usually not severe, and vomiting is uncommon. On the other hand such manifestations are prominent as a result of displacement of intracranial structures. ${ }^{10}$

Vomiting presented varying features, and even showed changes in the same patient during the course of the disease. In most of them, they occurred after meals and were sometimes preceded by nausea. One patient had morning vomiting related to postural changes. This feature, in association with dizziness, has been described in two patients in whom posterior fossa tumours were found. ${ }^{11}$ All our patients experienced weight loss and a deterioration of general health, features that should help differentiate it from "psychogenic" vomiting ${ }^{12} 13$ a frequent misdiagnosis after negative gastrointestinal investigations.

Vomiting is rarely a manifestation of epilepsy, and it then occurs mostly in children. ${ }^{14}$ It is usually episodic, paroxysmal, and stereotyped, and is accom- 
panied by a minor degree of impairment of consciousness, automatic behaviour, or amnesia. In addition, a personal or family history of seizures and EEG abnormalities may be present. ${ }^{15}$

In conclusion, analysis of our cases stresses the clinical point that persistent, protracted vomiting, in the absence of obvious gastrointestinal pathology, should raise concern about a lesion in or around the fourth ventricle, thus prompting appropriate diagnostic studies without delay.

The authors are grateful to Carlos S Kase, MD (Boston University, Boston, Massachusetts, USA) for help and criticism during the preparation of the manuscript.

\section{References}

1 Hanson JS, McCallum RW. The diagnosis and management of nausea and vomiting: A Review. Am J Gastroenterol 1985;80:210-8.

2 Borison HL, Wang SC. Physiology and pharmacology of vomiting. Pharmacol Rev 1953;5:193-230.

3 Borison HL, Borison R, McCarthy LE. Phylogenic and neurologic aspects of the vomiting process. J Clin Pharmacol 1981;21:235-95.

4 Miller A, Wilson V. Vomiting center reanalyzed and electrical stimulation study. Brain Res 1983;270:154-8.

5 Borison HL, Borison R, McCarthy LE. Role of the area postrema in vomiting and related functions. Fed Proc 1984;43:2955-7.

6 Harding RK, Hungelholtz H, Keaney M, Kucharczyk J. Discrete lesions of the area postrema abolish radiation-induced emesis in the dog. Neurosci Lett 1985;53:95-100.

7 McLellan L, Park D. Failure to vomit in hereditary ataxia. Neurology 1973;23:725-8.

8 Mitchell WG, Greenwood RS, Messenheimer JA. Abdominal epilepsy: Cyclic vomiting as the major symptom of simple partial seizure. Arch Neurol 1983;40:251-2.

9 Mulder DW, Daly D, Bailey AA. Visceral epilepsy. Arch Intern Med 1954;93:481-93.

10 Langfitt TW. Increased intracranial pressure and the cerebral circulation. In: Youmans JR, ed. Neurological Surgery. 2nd ed. Philadelphia: WB Saunders Co 1982:876-8.

11 Drachman DA, Diamond ER, Hart CW. Posturally evoked vomiting. Association with posterior fossa lesions. Ann Otol Rhinol Laryngol 1977;86:97-101.

12 Swanson D, Swanson W, Huizenaga K. Persistent nausea without organic cause. Mayo Clin Proc 1976;51:257-62.

13 Wruble LD, Rosenthal RH, Webb WL. Psychogenic vomiting: A review. Am J Gastroenterol 1982;77:318-21.

14 Millichap JG, Lombrosso CT, Lenox WG. Cyclic vomiting as a form of epilepsy in children. Pediatrics 1955;15:705-14.

15 Jacome DE, Fitzgerald R. Ictus emeticus. Neurology 1982;32:209-12. 